\title{
Base on metabolomics:analysis of the internal causes of nutrient changes in Leymus chinensis at different harvest stage
}

wei Wang

Inner Mongolia Agricultural University https://orcid.org/0000-0003-2064-3794

Gentu Ge

Inner Mongolia Agricultural University

Tian rong Zhou

Inner Mongolia Agricultural University

Chun Chang

Chinese Academy of Agricultural Sciences Grassland Research Institute

\section{Qiang Yin}

Chinese Academy of Agricultural Sciences Grassland Research Institute

Yushan Jia ( $\nabla$ jys_nm@sina.com )

https://orcid.org/0000-0001-7655-9933

\section{Research article}

Keywords: Leymus chinensis, Harvest stage, Nutrition quality, Metabolomics, Evaluation method

Posted Date: August 28th, 2019

DOl: https://doi.org/10.21203/rs.2.13629/v1

License: (c) (i) This work is licensed under a Creative Commons Attribution 4.0 International License.

Read Full License 


\section{Abstract}

Background Leymus chinensis is one of most valuable forage on the native grassland for its higher nutrition content. But the local farmer often gained the worse Leymus chinensis hay due to the unfavourable harvest stage, the nutrition of Leymus chinensis was decreasing from jointing stage to solid stage fro its growth characteristics, and the results showed that the crude protein (CP) decreased and the fiber content increased which will caused the nutrition of forage reduced. However, the reason for the nutrient changes in Leymus chinensis has not yet explained at the metabolic level. Results In this research, the Leymus chinensis was harvested at two growth stage(jointing stage and solid stage).Metabolomics was used to analyze the expression changes of metabolites in the whole plant of Leymus chinensis at different harvest stage, and the metabolites were analyzed based on LC-MS/MS untargeted metabolomics. A total of 55 metabolic pathways and 119 metabolites were related to the nutritional change in Leymus chinensis. Most of the metabolites are involved in metabolic processes, including amino acid metabolism, carbohydrate metabolism and acid metabolism biosynthesis. Under the action of EBF1/2 and EIN3, cysteine participates in the ubiquitin-mediated protein degradation pathway. In solid stage, the increasing content of cysteine directly promotes the ubiquitin-mediated protein degradation rate, which is manifested reduce the synthesis of crude protein (CP) in Leymus chinensis, resulting in the decreasing of Leymus chinensis nutrient quality. Carbohydrate metabolism provides raw materials for the synthesis of hemicellulose, leading to the increasing of hemicellulose. In addition, phenylalanine provides the necessary conditions for the synthesis of lignin. These are the internal factors that lead to the decline of quality of Leymus chinensis. Conclusions This study analyzed the elucidate the relationship between the reduction in the nutritional value of Leymus chinensis and complex biological processes by the metabolomic,This study will provides a theoretical basis for producing high-quality Leymus chinensis hay and sets the stage for further research.

\section{Background}

Leymus chinensis is a high quality grass of rhizome type belonging to the family of grasses[1]. Making hay is the main way of Leymus chinensis, which grows on the natural grassland. Because of its high nutritional value the fact that animals like to eat it when it is green as hay [2]. However, the value of Leymus chinensis hay is affected by lots of factors, the one was the harvest stage[3]. Leymus chinensis harvested at the jointing stage which has a greater nutrition than solid stage. At the tine of solid stage, the stem fraction of Leymus chinensis is greater than jointing stage. Many reporters have showed that decreased crude protein (CP) and increased fibre contents in Leymus chinensis will show different results at different stages[4]. Leymus chinensis harvest at the optimum growth stage can improve both hay yield and quality. The current view was harvested at the jointing stage is thought to cause a high yields and nutrient concentrations in Leymus chinensis. However, it is a difficult case in China especially in prairie areas, where Leymus chinensis is always harvested at solid stage due to the local farmer thought that more grass could be harvested at that time.However, due to the growth characteristics of plants, the Leymus chinensis harvested at solid stage had severe fibrosis and low nutritional value[5]. Lot of nutrients and relative metabolites, especially proteins, may decrease a lot during this time, which is 
regarded as a main index for evaluating the nutritional value of forage, indicating a large impact on the production of high-quality hay and the stable and sustainable development of grass husbandry[6].

We find out the specific changes in nutrients during this period by metabolomics, and metabolomics provide an useful methods for identifying metabolites and associated pathways that are crucial for understanding the nutrient contents of Leymus chinensis and the mechanisms underlying nutrient metabolite changes during different harvest stages[7]. At present, the research of metabolomics mainly focuses on the resistance,yield and nutrition of forage. There are few studies on the inner mechanism of using metabolomics to study the difference of nutrient quality of forage at different harvest stage. Metabolomics can promote the expression and identification of forage and its related metabolites. Metabolomics is an effective method for the analysis and identification of metabolites and metabolic pathways that may lead to decreased CP content in Leymus chinensis by LC-MS /MS [8].

At present, the evaluation methods of the nutritional value of forage mainly focuses on the index of $\mathrm{CP}$ and RFV[9]. The nutritional value of forage is mainly expressed by $\mathrm{CP}$, and the amount of $\mathrm{CP}$ will directly affect the nutritional value of forage[10]. Amino acids are important precursors in protein synthesis and have important significance for protein synthesis. Using metabolomics to study the specific pathways and metabolites of CP synthesis plays an important role in establishing a new grade of forage nutrient quality. In this study, using metabolomics to study changes in the nutritional value of forage will set a precedent for re-evaluating the nutritional value of forage.

In this research, We will use metabolic to fully studied protein synthesis pathways and metabolites, and our goal is to fully understand the nutrient changes, metabolites and metabolic pathways of Leymus chinensis. We also want to identify the key regulatory pathways and metabolites that influence changes in metabolites and nutrient content in Leymus chinensis.

\section{Statistical Analysis}

Three biological replicates were performed for each experiment, and nutritional data were analyzed using SAS 9.1.The mean differences were compared using Duncan's multiple range T-test. Comparisons with $P$ $<0.05$ were considered significantly significant, and the data are presented as the mean \pm SE, three independent replicates at least [11] .

\section{Results}

\section{Leymus chinensis nutrient content analysis}

In order to assess the composition of the Leymus chinensis at different harvest stage,the important nutritional content were listed ( $\mathrm{Tab} 1$ ). According to analyze of $\mathrm{CP}$, it show the highest content was existed the jointing stage, at $12.31 \% \mathrm{DM}$, and the lowest in the solid stage, at $8.27 \% \mathrm{DM}$. These results revealed that the CP content of Leymus chinensis continues to decreased from thejointing stage to solid stage. In contrast, the ADF content was $37.36 \%$ DM in jointing stage, which was significantly lower than the solid 
stage $(P<0.05)$. The content of NDF at jointing stage was $56.04 \% \mathrm{DM}$, which was significantly lower than at the solid stage $(P<0.05)$. The data showed that the fiber content in Leymus chinensis wasincreased gradually with the prolongation of growth period.

\section{Metabolic pathways associated with changes in protein content in Leymus chinensis}

To assess the metabolic pathways of the Leymus chinensis at different harvest stage,the main and secondary metabolism related to the change of CP in Leymus chinensis were analyzed and listed (Fig 1, Fig 2). we can find that the main metabolism contained glycan biosynthesis and metabolism, metabolism of terpenoids and polyketides, xenobiotics biodegradation and metabolism, lipid metabolism, carbohydrate metabolism, amino acid metabolism, enrgy metabolism, metabolism of other amino acid, nucleotide metabolism, metabolism of cafactours and vitamins, biosynthesis of secondary metabolism.meanwhile, the secondary metabolism concluded metabolism of terpenoids and polyketides, biosynthesis of cofactor and vitamins, and biosynthesis of other secondary metabolites.

\section{Metabolic pathways associated with protein change in Leymus chinensis at different harvest stage}

The Leymus chinensis were assessed by metabolomics from two harvest stages. A total of 119 metabolites of leymus chinensis were screened and identified, among which 64 metabolites were significantly up-regulated and 55 were significantly down-regulated(Table 2). After further study, we found that out of 55 down-regulated metabolic pathways were associated with protein reduction included 13 amino acids, 1 pyrimidine, 1 purine, 5 carbohydrate metabolism, 12 acid metabolism, and 23 other metabolism were involved(Tab 3).13 amino acids metabolic pathways included amino sugar and nucleotide sugar metabolism,cysteine and methionine metabolism, phenylalanine,tyrosine and tryptophan biosynthesis, phenylalanine metabolism, asparate and glutamate metabolism, clycine, serine and threonine metabolism, arginine and proline metabolism, arginine metabolism, lysine biosynthesis, lysine degradation, tyrosine metabolism, tryptophan metabolism, histidine metabolism; 5 carbohydrate metabolism included pentose phosphate pathway, galactose metabolism, fructose and mannose metabolism, glycolysis/gluconeogenesis, pentose and glucuronate interconversions; 12 acid metabolism included a-linolenic acid metabolism, C5-branched dibasic acid metabolism, 2-oxocarboxylic acid metabolism, biosynthesis of unsturated fatty acids, arachidonic acid metabolism, linoleic acid metabolism, nicotinate and nicotinamide metabolism, folate biosynthesis, glyoxylate and dicarboxylate metabolism, fatty acid metabolism, fatty acid biosynthesis, fatty acid elongation, fatty acid degradation. 23 other metabolism included terpenoid backbone biosynthesis, vancomycin resistance, and vitamin B6 and so on.

\section{Protein profiles of Leymus chinensis at different harvest stages}


This approach assisted in the accurate selection of marker metabolites and the investigation of changes in related metabolic processes (Fig. 3).Finally, 119 significant variations in metabolites were detected, which are shown in Table 2. These metabolites mainly included amino acids, organic acids, carbohydrates, purines, lipids and pyrimidines ( $\operatorname{Tab} 3$ ). A total of 55 metabolites were up-regulated while 64 were down-regulated at two harvest stages. The expression levels of L-glutamic acid, L-asparagine, purine, pyrimidine and other protein synthesis-related metabolites were down-regulated, whereas Lphenylalanine and carbohydrates, lipids and other substances were significantly up-regulated.

\section{Leymus chinensis hormone signal transduction}

There were eight signal transductiones was associate with the $\mathrm{CP}$ reduce in the leymus chinensis. The change of protein content in herbage is directly related to the metabolic reaction of amino acid. We can find there were three animo acid hormone signal transductiones( Fig 4), and they were tryptophan metabolism, cysteine and methonine metabolism, and phenyalalanine metabolism. There were two transduction in the the tryptophan metabolism, when the synthesis produce the AUXIAA, the one signal transduction reaction is ubiquitin mediated proteolysis, and the other signal transduction reaction was promote the cell enlargement, and it was helpful with the plant growth.Cysteine and methonine metabolism had two hormone signal transduction. After the EIN3 was existed, The one hormone signal transduction was to promote the ubiquitin mediated proteolysis, and the other signal transduction promote the fruit ripening senescence with the ERF1/2 and DNA.Phenyalalanine metabolism had one signal transduction, when the PR-1was existed, it was mainly used for disease resistance.

\section{Discussion}

\section{Metabolomics study of Leymus chinensis}

The CP content of forage is often different due to different species, harvest stage and other factors[12]. According to the chemical properties of forage, CP are mainly divided into two types[13]: true protein nitrogen and non-protein nitrogen. Non-protein nitrogen is mainly composed of free amino acids, amides, purines, pyrimidines and alkaloids, accounting for about $30 \%$ of the total nitrogen content of forage[14].Protein composition in foragemainly includes 20 amino acids, such as alanine, lysine, glutamic acid and aspartic acid. Amino acids, as the components of proteins, play a very important role in the nitrogen metabolism pathway in forage, and they are the preconditions for the composition of amino acids, purines and pyrimidines[15]. Studies have shown that glutamate plays a significant or extremely significant role in protein synthesis in wheat[16]. Study[17] showed that the content of glutamate in alfalfa in the early flowering stage was significantly higher than that in the mid-flowering stage $(P<0.05)$, and the L-glutamic acid was the main aimo acid which affect the CP changed. The above studies indicate that amino acids are related to protein synthesis, and the amount of amino acid content will affect the amount of protein synthesis, according to the results of this study alanine content in jointing stage is lower than that in solid stage. In addition, the levels of purine and pyrimidineare was lower in solid stage. 
Alanine, purines and pyrimidines content was reduced before the protein synthesis, which could lead to protein synthesis decreased, which was the reason of CP content in solid stage is lower than the jointing stage. The content of phenylalanine increased, but the protein content did not increase in the related metabolic pathway[18]. It can be considered that phenylalanine mainly comes from the hydrolysis of protein, which may also be one of the reasons for the decrease of protein content. Ttyrosine, after hydroxylation of phenylalanine, is involved in the process of glucose metabolism of plants was together with phenylalanine to promote the synthesis of carbohydrate[19]. The results of this study showed that the expression levels of sugars and other substances in solid stage were significantly up-regulated. Forage signaling figure of this study show that cysteine metabolic pathways in the generated EIN3, under the action of EBF1/2 and u, products are mainly used for ubiquitin protein degradation mediated, this is the only one in the plant in the metabolic pathway of protein degradation pathway, which may be CP in Leymus chinensis in jointing stage processed to the solid is the main reason, which will be our main research direction in the future.

The growth and development process of Leymus chinensis is mainly dominated by amino acid metabolism[20]. Amino acid, as the main mode of existence and transportation of nitrogen compounds in Leymus chinensis, is also regarded as the principle of maintaining the balance of nitrogen and the synthesis of enzymes and hormones in vivo[21]. The nutritional quality of Leymus chinensis relies mainly on the protein content, amino acid composition and its equilibrium state, amino acid content that in the condition with higher and lower which will affect the amount of protein synthesis, and the essential amino acid was considered as one of Leymus chinensis nutrition qualitative decision factors, on how to improve protein content at the same time, and the amino acid related research is a top priority in the current scholars research [22-24]. Studies have shown that the improvement of crop quality cannot rely solely on the simple accumulation of protein and amino acid content, but it should be comprehensively evaluated in combination with the yield of crops, especially the proportion of essential amino acids[25]. In the nitrogen metabolism pathway, the first is the assimilation of ammonia, followed by the absorption of amino acid nitrogen to participate in the nitrogen metabolism[26]. The assimilation of ammonia first forms glutamine and glutamic acid, and then forms other amino acids and proteins[27]. Glutamic acid plays an important role in the nitrogen metabolism pathway, because glutamic acid is the initial precursor of protein synthesis, which is mainly co-synthesized by alpha-ketoglutaric acid and amino acid transaminase[28]. In this study, the reduced content of glutamic acid and protein synthesis precursor decreased, which reduces the amount of protein synthesis in Leymus chinensis, performance for protein content is reduced, the main reason is that glutamate content directly to reduce the influence to the Leymus chinensis metabolism, influenced the late protein synthesis, and made an impact on the quality of the Leymus chinensis.

\section{Nutrition changes}

\section{Changes in nutrition (CP,ADF and NDF) contents changes}


In this study, harvest time affected CP of Leymus chinensis. Significant $(P<0.05)$, CP of Leymus chinensis decreased with the delay of harvest time. The decrease of CP in Leymus chinensismay was related to its growth and development, with the delay of harvest time, the ratio of stem and leaf of Leymus chinensis increased, while a large amount of CP in Leymus chinensis is stored in leaves, so the $\mathrm{CP}$ decreases. On the other hand, harvest time delayed will cause Leymus chinensis consume a large amount of $\mathrm{CP}$ for its own growth and the formation of awn needles, leading to the reduction of $\mathrm{CP}[29]$. The content of ADF and NDF has an important influence on its nutritional quality, among which ADF affects the digestibility[30]. NDF affects grazing rate of livestock, while high NDF content leads to low grazing rate[31]. In this study, harvest stage had significant effect on ADF and NDF of Leymus chinensis $(P<0.05)$. The content of ADF and NDF in Leymus chinensis increased with the delay of harvest stage, which may lead to the decrease of quality of Leymus chinensis with the delay of harvest stage.

In recent years, the proper harvesting of forage has been paid more and more attention by researchers at home and abroad. At present, the researches mainly focuses on the influence of harvest time on grass yield, quality and regeneration rate [32]. Some scholars pointed out that the proper harvest of forage should meet the following basic conditions[33]: first, the harvest stage should be conducive to the growth of forage; Secondly, it is necessary to maintain high nutritional quality of forage after harvest. Finally, harvesting is beneficial to the enrichment of nutrients in the roots of forage, so as to ensure the safe wintering of forage. Some scholars found that dry matter yield increased by $18 \%$ when alfalfa was cut at the peak of flowering, but dry matter digestibility and CP content decreased by $5.4 \%$ and $8.0 \%$ respectively[34]. With the prolongation of alfalfa growth period, the grass yield and dry matter content have been increasing, but the nutrients show a change trend of first increasing and then decreasing, and the budding stage is the critical point for the rise and fall of nutrients in forage[35]. When the forage was harvested at the flowering stage, the CP content remained th the highest level, and a higher yield could be obtained[36]. The above research results indicate that the harvest stage has an important influence on the preservation of forage's nutritional quality. During the whole growth period of native grass, with the continuous extension of the growth period of forage, the forage yield gradually increases, but the quality gradually decreases. The longer the harvest period, the higher fiber. Therefore, grass yield and nutrient quality should be taken into account in the harvest of forage. Harvest stage has a great impact on the yield, quality and rejuvenation of forage, and proper harvest can ensure the yield and nutritional value of forage.

\section{Establishment of forage nutrition evaluation methods}

At present, the CP, NDF and ADF are the common indicators to evaluate nutritive value while the $\mathrm{CP}$ is the main index, but the studies about the quality evaluation of forage from the level of metabolism has not mentioned. Due to the physiological characteristics of forage heterogeneity, the different harvest stage will cause the different CP. In order to get a higher CP, we need to choose the suitable harvest stage[37]. $\mathrm{CP}$ was mainly expressed on the physiological and biochemical reaction synthesis of amino acids, and alanine as an important precursor of protein synthesis, which has important influence on the formation of 
a protein, and we can say alanine content on the forage quality has important influence, and this experiment showed alanine was important on the influence of $\mathrm{CP}$, to explained the amino acids from molecular level for the importance of forage quality evaluation[38]. Therefore, based on the analysis results of this experiment, it is necessary to continue to clarify the changes of forage quality through amino acid molecules such as glutamic acid, strengthen the research on the relationship between protein synthesis precursor and forage quality, and discuss the evaluation index of hay quality from the molecular level.

\section{Conclusions}

Our data analyzed and interpretated the Leymus chinensis metabolites, the change of metabolic pathways, and the aspects of nutritional quality changes on Leymus chinensis metabonomicson from jointing stage to the solid stage. A large number of different expressed metabolites are mainly involved in carbohydrate expression metabolism, starch and sucrose metabolism, cysteine biosynthesis and amino acid biosynthesis. Leymus chinensis has low CP in solid stage, mainly due to glutamic acid content decreased. The metabolism of carbohydrate synthesis of hemicellulose do not receive the raw material, cause the grass hemicellulose content increases, further lead to increased NDF content. In addition, the increase of phenylalanine content of lignin synthesis provides the necessary conditions. Together, these results provided the internal causes of the content of CP decreased and NDF and ADF increased in Leymus chinensis from jointing stage to the solid stage. This study provides the result of the innovation and further clarified on the quality difference of Leymus chinensis at two important stages. In addition, it offers a new methods to explain and evaluate forage nutrition, and our results also show the reasons for $\mathrm{CP}$ changes. Therefore, this study illustrates the nutritional value of Leymus chinensisreduce relation with complex metabolic biological process, and provide the theoretical basis for the future production and technical support of Leymus chinensis.

\section{Abbreviations}

CP:crude protein

NDF: neutral detergent fiber

ADF: acid detergent fiber

QC:quality control

\section{Methods}

\section{Forage material}

Leymus chinensis was harvested at Inner Mongolia Agricultural University field experimental site in Chifen $\left(41^{\circ} 17^{\prime} 10^{\prime \prime} \varangle 45^{\circ} 24^{\prime} 15^{\prime \prime} \mathrm{N} \otimes 116^{\circ} 21^{\prime} 07^{\prime \prime} \otimes 120^{\circ} 58^{\prime} 52 " \mathrm{E}\right)$, Inner Mongolia, China, in 2018 . We are licensed by 
the local agriculture and pastoral bureau and certified by grass industry experts, and we are conducting experiments in accordance with the local grassland protection law. the voucher specimen of the Leymus chinensis material has been deposited in Operation department of hangzhou lianchuan biotechnology co., LTD, the company was located in 4th floor, building 16, zhongzi science and technology park, no. 260, xiasha 6th avenue, hangzhou economic and technological development zone (310018), and the deposition number of Leymus chinensis was $2018 \mathrm{H} 27 \mathrm{mqgA} 82$. The selected Leymus chinensis is naturally grown on native grassland. When taking samples of Leymus chinensis, the staff followed the local grassland protection law and the regulations on sampling wild species in the convention on trade in endangered species of wild fauna and do as the :https://www.cites.org/ requested. The identification of Leymus chinensis samples was conducted after consulting with Mr Zhang weiguo, a well-known expert in plant classification in China. Zhang weiguo devoted himself to the identification and classification of forage grass species in the field all the year around, with rich field work experience and scientific research foundation. We took Leymus chinensis as the experimental material and started the experiment on August 20,2017 . When the forage was harvested, the growth periods were jointing stage and solid stage. The jointing stage is a period which has a rapid growth of stem on Leymus chinensis. Generally, the first stem node of more than $50 \%$ plants in the whole field is exposed to the ground by $1.5 \varangle 2.5 \mathrm{~cm}$, and solid stage refers to the period from flowering to maturity of forage seeds. We took samples with sterilized gloves and cut them with sterilized scissors to gained two stage samples. Each sample was $200 \mathrm{~g}$, and one sample was placed in an envelope bag, then the samples were dried in a $105^{\circ} \mathrm{C}$ oven for $30 \mathrm{~min}$. Washing the Leymus chinensis samples with PBS buffer, wraping them with tin foil, marking them and immediately freeze them in liquid nitrogen, and then transfer them to $-80^{\circ} \mathrm{C}$ refrigerator for later use.

\section{Nutrition Analyze}

We analyzed the crude protein (CP) in accordance with the reference of the AOAC international [39]. The content of neutral detergent fiber (NDF) and acid detergent fiber (ADF) were analyzed according to the Ankom A200 fiber analyzer (Ankom Technology, Macedon, NY) by the method of Van Soest[40].

\section{Metabolite Analyze}

\section{Description of metabolite extraction}

Samples were defrosted on ice and metabolites were extracted with $50 \%$ methanol buffer. 20 mul samples were used to extract 120 mul $50 \%$ methanol and incubated at room temperature for 10 minutes. The extract was stored overnight at $-20^{\circ} \mathrm{C}$.. After centrifugation at $4,000 \mathrm{~g}$ for $20 \mathrm{~min}$, the supernatants were placed into new 96 -well plates. The samples were placed at $-80^{\circ} \mathrm{C}$ prior to the LC-MS analysis. In addition, pooled QC samples were also prepared by combining $10 \mu \mathrm{L}$ of each extraction mixture.

\section{Liquid phase parameter description}


All experimental samples were acquired by the LC-MS system followed machine orders. First, all chromatographic separations were performed using an ultra performance liquid chromatography (UPLC) system (SCIEX, UK). One ACQUITY UPLC T3 column which was $100 \mathrm{~mm} \star 2.1 \mathrm{~mm}, 1.8 \mu \mathrm{m}$, Waters, UK and it was worked for the reversed phase separation. The column oven was kept at $35^{\circ} \mathrm{C}$. The flow rate was maintained at $0.4 \mathrm{ml} / \mathrm{min}$ and the mobile phase composite of solvent $A$ and solvent $B$, the solvent $A$ was water, $0.1 \%$ formic acid while the solvent $B$ was acetonitrile, $0.1 \%$ formic acid.

\section{Mass spectrum parameter description}

We detect metabolites by a high-resolution tandem mass spectrometer TripleTOF5600plus (SCIEX, UK), and the Q-TOF was operated at two styles of positive and negative.

And we set the curtain gas $30 \mathrm{PSI}$, the ion source gas $160 \mathrm{PSI}$, the ion source gas2 $60 \mathrm{PSI}$, and the temperature of interface heater was $650{ }^{\circ} \mathrm{C}$.

For the positive ion mode, the floating voltage of ion spray is set to $5000 \mathrm{~V}$ respectively. For the negative ion mode, the floating voltage of ion spray is set to $-4500 \mathrm{v}$ respectively. The mass spectrometry data were collected by IDA mode. The mass of the TOF ranges from 60 to $1200 \mathrm{Da}$. Survey scans are taken within 150 milliseconds, and up to 12 product ion scans will be collected if the threshold of 100 counts per second (count per second) is exceeded and the 1+ charging state is in place. The total cycle time was fixed at $0.56 \mathrm{~s}$. By monitoring the $40 \mathrm{ghz}$ multi-channel TDC detector (four-anode/channel detection), four time boxes were calculated for each scan with a pulse frequency value of $11 \mathrm{khz}$. Dynamic exclusion is set to 4 seconds. During the collection process, quality accuracy is calibrated for every 20 samples. In addition, to evaluate the stability of Ic-ms throughout the collection process, one quality control sample (all sample pools) was collected after every 10 samples.

Information analysis description

The acquired MS data pretreatments including peak picking, peak grouping, retention time correction, second peak grouping, and annotation of isotopes and adducts was performed using XCMS software. LC-MS raw data files were converted into $\mathrm{mzXML}$ format and then processed by the XCMS, CAMERA and metaX toolbox implemented with the $\mathrm{R}$ software. Each ion was identified by combining retention time (RT) and $\mathrm{m} / \mathrm{z}$ data. Intensities of each peaks were recorded and a three dimensional matrix containing arbitrarily assigned peak indices (retention time-m/z pairs), sample names (observations) and ion intensity information (variables) was generated.

The online KEGG, HMDB database was used to annotate the metabolites by matching the exact molecular mass data $(\mathrm{m} / \mathrm{z})$ of samples with those from database. If a mass difference between observed and the database value was less than $10 \mathrm{ppm}$, the metabolite would be annotated and the molecular formula of metabolites would further be identified and validated by the isotopic distribution measurements. We also used a in-house fragment spectrum library of metabolites to validate the metabolite identidification. 
The intensity of peak data was further preprocessed by metaX. Those features that were detected in less than $50 \%$ of QC samples or $80 \%$ of biological samples were removed, the remaining peaks with missing values were imputed with the k-nearest neighbor algorithm to further improve the data quality. PCA was performed for outlier detection and batch effects evaluation using the pre-processed dataset. Quality control-based robust LOESS signal correction was fitted to the QC data with respect to the order of injection to minimize signal intensity drift over time. In addition, the relative standard deviations of the metabolic features were calculated across all QC samples, and those $>30 \%$ were then removed.

Student t-tests were conducted to detect differences in metabolite concentrations between 2 phenotype.The P value was adjusted for multiple tests using an FDR (Benjamini-Hochberg). Supervised PLS-DA was conducted through metaX to discriminate the different variables between groups. The VIP value was calculated. A VIP cut-off value of 1.0 was used to select important features.

\section{Declarations}

\section{Ethics approval and consent to participate}

Not applicable

\section{Consent to $>$ publish}

Not applicable

\section{Availability of data and materials}

All data generated or analyzed during this study are included in this published article and its supplementary information files.

\section{Competing Interest}

The authors declare that they have no conflicts of interest to disclose.

\section{Funding}

This study was supported by "Study on Research and Development of Hay Production with Low Loss and High Quality Demonstration" (2017YFD0502103), Ministry of Science and Technology, China and project "China Agriculture Research System" (CARS-34), Ministry of Agriculture.

\section{Authors' contributions}

WW, GGT and YSJ designed the experiments; WW and TRZ conducted the experiments; WW, CC and QY analyzed the data; WW and TRZ wrote the paper. All authors read and approved the final manuscript. 
We are thankful for the assistance provided by Wenqiang-Fan for technical support. Thank American Journal Experts (AJE) language editing service for language edit

\section{References}

1. 1 Afkhami Michelle E., Luke Mahler D., Burns Jean H., et al. Symbioses with nitrogen-fixing bacteria: nodulation and phylogenetic data across legume genera. Ecology, 2017, 99(2): 502-502.

2. 2 Bai Y., Wu J., Clark CM., et al. Tradeoffs and thresholds in the effects of nitrogen addition on biodiversity and ecosystem functioning: evidence from inner Mongolia Grasslands. Global Change

3. Biology, 2010, 16(1): 358-372.

4. 3 Wood C D, Manyuchi B.Use of an in vitro gas production method to investigate interactions between veld hay and Napier hay or groundnut hay supplements.Anim Feed Sci Tech, 1997, 679:265278.

5. 4 Yari M, Valizadeh R, Naserian AA, Jonker A, Yu P. Modeling nutrient availability of alfalfa hay harvested at three stages of maturity and in the afternoon and morning in dairy cows. Anim Feed Sci Tech. 2012;178(1):9-12.

6. 5 SHRIVASTAVA P, KUMAR R.Soil salinity:A serious environmental issue and plant growth promoting bacteria as one of the tools for its alleviation.Saudi Journal of Biological Sciences, 2015, 22 (2):123131.

7. 6 Boot R.G.A., Mensink M. Size and morphology of root systems of perennial grasses from contrasting habitats as affected by nitrogen supply. Plant and Soil, 1990, 129(2): 291-299.

8. 7 Smith CA, Want EJ, O'Maille G, Ruben Abagyan A, Siuzdak G. XCMS: processing mass spectrometry data for metabolite profiling using nonlinear peak alignment, matching, and identification. Anal Chem. 2006;78(3):779.

9. 8 Lamb JFS, Sheaffer CC, Samac DA. Population density and harvest maturity effects on leaf and stem yield in alfalfa this paper is a joint contribution from the plant Sci. Res. Unit, USDA-ARS, and the Minnesota Agric. Exp. Stn. Agron J. 2003;95(3):635-41.

10. 9 Chapin III F.S. The mineral nutrition of wild plants. Annual Review of Ecology \& Systematics, 1980, 11(1): 233-260.

11. 10 Bai Y., Wu J., Clark C.M., et al. Grazing alters ecosystem functioning and C:N:P stoichiometry of

12. grasslands along a regional precipitation gradient. Journal of Applied Ecology, 2012, 49(6): 12041215. 11 SAS. SAS user's guide: Statistic Analysis System, Version 9.1. SAS Institute Inc., Cary, NC, USA. 2003

13. Campbell W.H. Nitrate reductase structure, function and regulation 囚bridging the gap between biochemistry and physiology. Annual Review of Plant Physiology and Plant Molecular Biology, 1999, 50(1): 277-303.

14. Lamb JFS, Jung H-JG, Riday H. Growth environment, harvest management and germplasm impacts on potential ethanol and crude protein yield in alfalfa. Biomass Bioenergy. 2014;63:114-25. 
15. Popescu GV, Noutsos C, Popescu SC. Big data in plant science: Resources and data mining tools for plant genomics and proteomics. Methods in Molecular Biology, 2016, 1415:533-543.

16. Yagi Y, Shiina T. Recent advances in the study of chloroplast gene expression and its evolution. Frontiers in Plant Science, 2014, 5(1):61-72.

17. Cease A.J., Elser J.J., Ford C.F., et Heavy livestock grazing promotes locust outbreaks by lowering plant nitrogen content. Science, 2012, 335(6067): 467.

18. Fan WQ , Ge GT , Liu YH, et al.Proteomics integrated with metabolomics: analysis of the internal causes of nutrient changes in alfalfa at different growth stages.BMC Plant Biology. 2018, 18:78

19. Yuan XJ, Yu CQ, Li ZH, et al. Effect of inclusion of grasses and wet hulless barley distillers' grains on the fermentation and nutritive quality of oat straw and straw-grass silages in Tibet. J Anim Produc Sci 2013; 53:419-26.

20. Chapin III F.S., Shaver G.R. Individualistic growth response of tundra plant species to environmental manipulations in the field. Ecology, 1985, 66(2): 564-576.

21. Fort F., Cruz P., Jouany C. Hierarchy of root functional trait values and plasticity drive early-stage competition for water and phosphorus among grasses. Functional Ecology, 2014, 28(4): 1030-1040.

22. McDonald I. A revised model for the estimation of protein degradability in the rumen. J Agr Sci 1981; 96:251-2.

23. 22 Hill J.O., Simpson R.J., Moore A.D., et al. Morphology and response of roots of pasture species to

24. phosphorus and nitrogen nutrition. Plant and Soil, 2006, 286(1): 7.

25. 23 Barnett NM, W haylor. Amino acid and protein metabolish in Bermuda grass during water stress. Plant Physiol.1996,41:1222-1230.

26. 24 Kramer-Walter K.R., Laughlin D.C. Root nutrient concentration and biomass allocation are more plastic than morphological traits in response to nutrient limitation. Plant and Soil, 2017, 416(1): 539550.

27. 25 Adams E.Metabolism of proline and hydroxy Proline. Ann.Rev.Bioehem.,1980;49:1005-1061

28. 26 Wang L, Pan D, Jian L, et al. Proteomic analysis of changes in the Kandelia candel, chloroplast proteins reveals pathways associated with salt tolerance. Plant Science, 2015, 231:159-172.

29. 27 Lü X., Freschet G.T., Kazakou E., et al. Contrasting responses in leaf nutrient-use strategies of two 30. dominant grass species along a 30-yr temperate steppe grazing exclusion chronosequence. Plant and Soil, 2015, 387(1): 69-79.

31. 28 Lam H.M., Coschigano K.T., Oliveira IC., et al. The Molecular-genetics of nitrogen assimilation into amino acids in higher plants. Annual Review of Plant Physiology and Plant Molecular Biology, 1996, 47(1): 569-593.

32.

33.

34. 29 SHRIVASTAVA P, KUMAR R.Soil salinity:A serious environmental issue and plant growth promoting bacteria as one of the tools for its alleviation.Saudi Journal of Biological Sciences, 2015, 22 (2):123- 
131.

35. 30 Grant RJ, Weidner SJ. Digestion kinetics of fiber: influence of in vitro buffer $\mathrm{pH}$ varied within observed physiological range. J Dairy Sci 1992; 75:1060-8.

36. 31 Lamont B. Mechanisms for enhancing nutrient uptake in plants, with particular reference to mediterranean South Africa and Western Australia. The Botanical Review, 1982, 48(3): 597-689.

37. 32 BORK EW, BROADBENT TS, WILLMS WD. Intermittent growing season defoliation variably impacts accumulated herbage productivity in mixed grass prairie. Rangeland Ecology and Management, 201770 (3) :307-315.

38. 33 HUME DE. Effect of cutting on production and tillering in Prairie Grass (Bromus willdenowii Kunth) compared with two Ryegrass (Lolium) species. 2. reproductive pants. Annals of Botany, 1991, 67:533-541.

39. 34 Li X., Hou X., Ren W., et al. Long-term effects of mowing on plasticity and allometry of Leymus 40. chinensis in a temperate semi-arid grassland, China. Journal of Arid Land, 2016, 8(6): 899-909.

41. 35 Aranjuelo I, Molero G, Erice G, Avice JC, Nogués S. Plant physiology and proteomics reveals the leaf response to drought in alfalfa (Medicago sativa L.). J Exp Bot. 2011;62(1):111.

42. 36 Li Y., Wang W., Liu Z., et al. Grazing gradient versus restoration succession of Leymus chinensis

43. (Trin.) Tzvel. grassland in Inner Mongolia. Restoration Ecology, 2008, 16(4): 572-583.

44.

45. 37 Pan Q., Bai Y., Han X., et al. Effects of nitrogen additions on a Leymus chinensis population in typical steppe of Inner Mongolia. Acta Phytoecological Sinica, 2005, 29(2): 311-317.

46. 38 Niu F, Duan D, Chen J, et al.Eco-Physiological Responses of Dominant Species to watering in a Natural Grassland Community on the Semi-Arid Loess Plateau of China[J].Frontiers in Plant Science, 2016 (7) :663-673

47. 39 Karam E A, Keramat B, Asrar Z, et al.Triacontanol-induced changes in growth, oxidative defense system in Coriander (Coriandrum sativum) under arsenic toxicity[J].Indian Journal of Plant Physiology, 2016, 21 (2) :137-142.

48. 40 Van Soest PJ, Robertson JB, Lewis BA. Methods for dietary fiber, neutral detergent fiber, and nonstarch polysaccharides in relation to animal nutrition. J Dairy Sci. 1991;74(10):3583-97.

\section{Tables}

Table 1 Nutrients of Leymus chinensis at different harvest stage

\begin{tabular}{cccc}
\hline Harvest stage & CP $\%$ DM $\square$ & ADF \% DM $\square$ & NDF $\%$ DM $\square$ \\
\hline Jointing stage & $12.31 \pm 0.14 \mathrm{a}$ & $37.36 \pm 0.36 \mathrm{~b}$ & $56.04 \pm 1.01 \mathrm{~b}$ \\
Solid stage & $8.27 \pm 0.77 \mathrm{~b}$ & $43.65 \pm 0.33 \mathrm{a}$ & $72.36 \pm 1.63 \mathrm{a}$ \\
\hline
\end{tabular}


$\mathrm{a}, \mathrm{b}$ Means $\pm \mathrm{SD}$ within columns with different letters indicating significant differences $(P<0.05)$

Table2 The changes of metabolites in Leymus chinensis 


\begin{tabular}{|c|c|c|c|c|}
\hline Number & $\begin{array}{l}\text { Model } \\
\text { up }\end{array}$ & Metabolites & $\begin{array}{l}\text { Model } \\
\text { down }\end{array}$ & Metabolites \\
\hline 1 & $\operatorname{ESI}(+)$ & (R)-3-Amino-2-methylpropanoate & $\operatorname{ESI}(-)$ & L-2-Aminoadipate \\
\hline 2 & $\operatorname{ESI}(+)$ & L-3-Aminoisobutanoate & $\operatorname{ESI}(-)$ & LL-2,6-Diaminoheptanedioate \\
\hline 3 & $\operatorname{ESI}(+)$ & 3-Methyl-2-oxobutanoic acid & $\operatorname{ESI}(-)$ & meso-2,6-Diaminoheptanedioate \\
\hline 4 & $\operatorname{ESI}(+)$ & L-Valine & $\mathrm{ESI}(-)$ & $\begin{array}{l}(2 \mathrm{~S}, 4 \mathrm{~S})-4 \text {-Hydroxy-2,3,4,5- } \\
\text { tetrahydrodipicolinate }\end{array}$ \\
\hline 5 & $\operatorname{ESI}(+)$ & Methylmalonate & $\operatorname{ESI}(-)$ & N-Acetyl-L-2-amino-6-oxopimelate \\
\hline 6 & $\operatorname{ESI}(+)$ & 2-Methyl-1-hydroxypropyl-ThPP & $\operatorname{ESI}(-)$ & N-Succinyl-LL-2,6-diaminoheptanedioate \\
\hline 7 & $\operatorname{ESI}(+)$ & 3-Methyl-1-hydroxybutyl-ThPP & $\operatorname{ESI}(-)$ & N6-(L-1,3-Dicarboxypropyl)-L-lysine \\
\hline 8 & $\operatorname{ESI}(+)$ & D-Xylose & $\operatorname{ESI}(-)$ & L-Glutamate \\
\hline 9 & $\operatorname{ESI}(+)$ & L-Arabinose & $\operatorname{ESI}(-)$ & L-Arginine \\
\hline 10 & $\operatorname{ESI}(+)$ & D-Ribulose & $\operatorname{ESI}(-)$ & N-Acetyl-L-glutamate 5-semialdehyde \\
\hline 11 & $\operatorname{ESI}(+)$ & D-Xylulose & $\operatorname{ESI}(-)$ & L-Leucine \\
\hline 12 & $\operatorname{ESI}(+)$ & L-Xylulose & $\operatorname{ESI}(-)$ & L-Isoleucine \\
\hline 13 & $\mathrm{ESI}(+)$ & D-Lyxose & $\mathrm{ESI}(-)$ & L-Glutamate \\
\hline 14 & $\mathrm{ESI}(+)$ & L-Ribulose & $\operatorname{ESI}(-)$ & O-Acetyl-L-serine \\
\hline 15 & $\mathrm{ESI}(+)$ & L-Lyxose & $\operatorname{ESI}(-)$ & L-2-Aminoadipate \\
\hline 16 & $\operatorname{ESI}(+)$ & Xylitol & $\operatorname{ESI}(-)$ & L-Arginine \\
\hline 17 & $\mathrm{ESI}(+)$ & Ribitol & $\operatorname{ESI}(-)$ & L-Phenylalanine \\
\hline 18 & $\operatorname{ESI}(+)$ & L-Arabitol & $\operatorname{ESI}(-)$ & LL-2,6-Diaminoheptanedioate \\
\hline 19 & $\operatorname{ESI}(+)$ & D-Arabitol & $\operatorname{ESI}(-)$ & meso-2,6-Diaminoheptanedioate \\
\hline 20 & $\operatorname{ESI}(+)$ & alpha'-Trehalose 6-phosphate & $\operatorname{ESI}(-)$ & N-Acetyl-L-glutamate 5-semialdehyde \\
\hline 21 & $\operatorname{ESI}(+)$ & Sucrose 6'-phosphate & $\operatorname{ESI}(-)$ & L-Histidine \\
\hline 22 & $\operatorname{ESI}(+)$ & Maltose 6'-phosphate & $\operatorname{ESI}(-)$ & $\begin{array}{l}\text { (2S } \square 4 \mathrm{~S})-4 \text {-Hydroxy-2,3,4,5- } \\
\text { tetrahydrodipicolinate }\end{array}$ \\
\hline 23 & $\operatorname{ESI}(+)$ & $\begin{array}{l}\text { 6-Phospho-beta-D-glucosyl-(1,4)-D- } \\
\text { glucose }\end{array}$ & $\operatorname{ESI}(-)$ & O-Phospho-L-serine \\
\hline 24 & $\operatorname{ESI}(+)$ & Sucrose 6-phosphate & $\operatorname{ESI}(-)$ & L-Tryptophan \\
\hline
\end{tabular}




\begin{tabular}{|c|c|c|c|c|}
\hline 25 & ESI(+) & alpha-Maltose 1-phosphate & $\mathrm{ESI}(-)$ & L-Arginine \\
\hline 26 & $\operatorname{ESI}(+)$ & Anthranilate & ESI $(-)$ & D-Arginine \\
\hline 27 & $\operatorname{ESI}(+)$ & 3-Hydroxybenzoate & ESI $(-)$ & L-Phenylalanine \\
\hline 28 & $\mathrm{ESI}(+)$ & 3,4-Dihydroxybenzoate & ESI $(-)$ & 2-Oxo-4-phenylbutyric acid \\
\hline 29 & $\operatorname{ESI}(+)$ & 2-Oxo-4-phenylbutyric acid & ESI $(-)$ & L-Tryptophan \\
\hline 30 & $\operatorname{ESI}(+)$ & L-Arogenate & ESI(-) & $\begin{array}{l}\text { 2-Amino-3, 7-dideoxy-D-threo-hept-6- } \\
\text { ulosonic acid }\end{array}$ \\
\hline 31 & $\mathrm{ESI}(+)$ & Shikimate 3-phosphate & ESI $(-)$ & 6-Deoxy-5-ketofructose 1-phosphate \\
\hline 32 & $\mathrm{ESI}(+)$ & 5-Amino-2-oxopentanoic acid & $\mathrm{ESI}(-)$ & L-Glutamate \\
\hline 33 & $\mathrm{ESI}(+)$ & 2-Amino-4-oxopentanoic acid & $\mathrm{ESI}(-)$ & 5-Phosphoribosylamine \\
\hline 34 & $\mathrm{ESI}(+)$ & Phenylacetaldehyde & $\mathrm{ESI}(-)$ & D-Glucosamine 6-phosphate \\
\hline 35 & $\operatorname{ESI}(+)$ & Succinate & $\mathrm{ESI}(-)$ & N-Acetylaspartylglutamate \\
\hline 36 & $\operatorname{ESI}(+)$ & Phenylacetic acid & $\mathrm{ESI}(-)$ & Ectoine \\
\hline 37 & $\mathrm{ESI}(+)$ & 4-Hydroxybenzoate & ESI $(-)$ & O-Phospho-L-serine \\
\hline 38 & $\mathrm{ESI}(+)$ & Salicylate & $\mathrm{ESI}(-)$ & L-Tryptophan \\
\hline 39 & $\mathrm{ESI}(+)$ & Phenylpropanoate & ESI $(-)$ & Tetrahydrofolate \\
\hline 40 & $\mathrm{ESI}(+)$ & alpha-Oxo-benzeneacetic acid & $\operatorname{ESI}(-)$ & 2,6-Dihydroxyphenylacetate \\
\hline 41 & $\mathrm{ESI}(+)$ & 4-Hydroxy-2-oxopentanoate & $\mathrm{ESI}(-)$ & L-Phenylalanine [ \\
\hline 42 & $\mathrm{ESI}(+)$ & $\begin{array}{l}\text { cis-3-(Carboxy-ethyl)-3,5-cyclo- } \\
\text { hexadiene-1,2-diol }\end{array}$ & ESI(-) & D-Phenylalanine \\
\hline 43 & $\operatorname{ESI}(+)$ & Lactose 6'-phosphate & $\operatorname{ESI}(-)$ & Phenylacetylglutamine \\
\hline 44 & $\mathrm{ESI}(+)$ & $\begin{array}{l}\text { 2-Dehydro-3-deoxy-6-phospho-D- } \\
\text { galactonate }\end{array}$ & $\mathrm{ESI}(-)$ & Hypoxanthine \\
\hline 45 & $\operatorname{ESI}(+)$ & 3-beta-D-Galactosyl-sn-glycerol & ESI $(-)$ & 5-Phosphoribosylamine \\
\hline 46 & $\mathrm{ESI}(+)$ & Melibiitol & $\mathrm{ESI}(-)$ & Inosine \\
\hline 47 & $\operatorname{ESI}(+)$ & Raffinose & $\mathrm{ESI}(-)$ & $\begin{array}{l}\text { 2-(Formamido)-N1-(5'- } \\
\text { phosphoribosyl)acetamidine }\end{array}$ \\
\hline 48 & $\mathrm{ESI}(+)$ & $\begin{array}{l}\text { D-Gal alpha 1->6D-Gal alpha 1->6D- } \\
\text { Glucose }\end{array}$ & ESI (-) & Urate-3-ribonucleoside \\
\hline
\end{tabular}




\begin{tabular}{|c|c|c|c|c|}
\hline 49 & $\operatorname{ESI}(+)$ & Stachyose & $\mathrm{ESI}(-)$ & Mannitol \\
\hline 50 & $\operatorname{ESI}(+)$ & Arbutin & $\mathrm{ESI}(-)$ & D-Sorbitol \\
\hline 51 & $\operatorname{ESI}(+)$ & (R)-3-Amino-2-methylpropanoate & ESI(-) & 2-O-(alpha-D-Mannosyl)-D-glycerate \\
\hline 52 & $\operatorname{ESI}(+)$ & 5-Methylcytosine & $\mathrm{ESI}(-)$ & D-Sorbitol \\
\hline 53 & $\operatorname{ESI}(+)$ & Thymine & ESI(-) & Galactitol \\
\hline 54 & $\operatorname{ESI}(+)$ & Methylmalonate & $\mathrm{ESI}(-)$ & D-Galactosamine 6-phosphate \\
\hline 55 & $\operatorname{ESI}(+)$ & Thymidine & $\mathrm{ESI}(-)$ & N-Acetyl-D-galactosamine \\
\hline 56 & $\operatorname{ESI}(+)$ & Adenine & & \\
\hline 57 & $\operatorname{ESI}(+)$ & Secologanin & & \\
\hline 58 & $\operatorname{ESI}(+)$ & 4,21-Dehydrogeissoschizine & & \\
\hline 59 & $\operatorname{ESI}(+)$ & 4,21-Dehydrocorynantheine aldehyde & & \\
\hline 60 & $\operatorname{ESI}(+)$ & Strictosidine aglycone & & \\
\hline 61 & $\operatorname{ESI}(+)$ & Horhammericine & & \\
\hline 62 & $\operatorname{ESI}(+)$ & Dialdehyde & & \\
\hline 63 & $\operatorname{ESI}(+)$ & Vindoline & & \\
\hline 64 & $\mathrm{ESI}(+)$ & Vinblastine & & \\
\hline
\end{tabular}

Table 3 Metabolic pathways associated with protein decline in Leymus chinensis 


\begin{tabular}{|c|c|c|}
\hline $\begin{array}{l}\text { Metabolic } \\
\text { pathway } \\
\text { items }\end{array}$ & Number & Metabolism \\
\hline \multirow[t]{7}{*}{ Amino acids } & 13 & Amino sugar and nucleotide sugar metabolism, Cysteine and methionine metabolism \\
\hline & & Phenylalanine, tyrosine and tryptophan biosynthesis Phenylalanine Metabolism \\
\hline & & Asparate and glutamate metabolism ,Clycine, serine and threonine metabolism \\
\hline & & Arginine and proline metabolism, Arginine metabolism \\
\hline & & Lysine biosynthesis ,Lysine degradation \\
\hline & & Tyrosine metabolism ,Tryptophan metabolism \\
\hline & & Histidine metabolism \\
\hline Pyrimidine & 1 & Pyrimidine metabolism \\
\hline Purine & 1 & Purine metabolism \\
\hline \multirow[t]{2}{*}{$\begin{array}{l}\text { Carbohydrate } \\
\text { metabolism }\end{array}$} & 5 & $\begin{array}{l}\text { Pentose phosphate pathway ;Galactose metabolism ;Fructose and mannose } \\
\text { metabolism;Glycolysis/gluconeogenesis }\end{array}$ \\
\hline & & Pentose and glucuronate interconversions \\
\hline \multirow[t]{2}{*}{ Acid } & 12 & $\begin{array}{l}\text { A-linolenic acid metabolism;C5-branched dibasic acid metabolism;2-Oxocarboxylic } \\
\text { acid metabolism ;Biosynthesis of unsturated fatty acids;Arachidonic acid } \\
\text { metabolism;Linoleic acid metabolism ;Nicotinate and nicotinamide metabolism }\end{array}$ \\
\hline & & $\begin{array}{l}\text { Folate biosynthesis ;Glyoxylate and dicarboxylate metabolism;Fatty acid metabolism } \\
\text {;Fatty acid biosynthesis;Fatty acid elongation, fatty acid degradation }\end{array}$ \\
\hline $\begin{array}{l}\text { Other } \\
\text { metabolism }\end{array}$ & 23 & Terpenoid backbone biosynthesis;Vancomycin resistance ;Vitamin B6 and so on \\
\hline
\end{tabular}

\section{Figures}




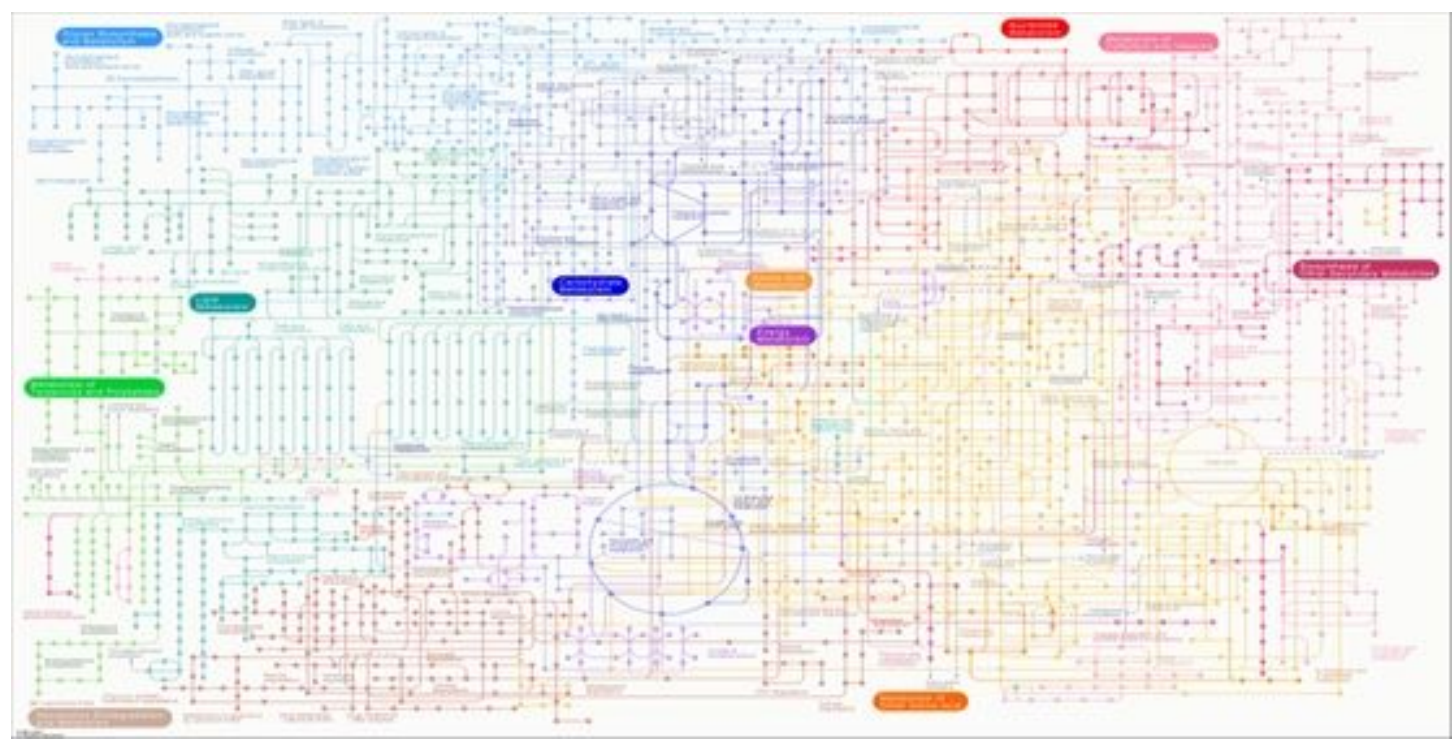

\section{Figure 1}

The main metabolism related to the change of CP content in Leymus chinensis

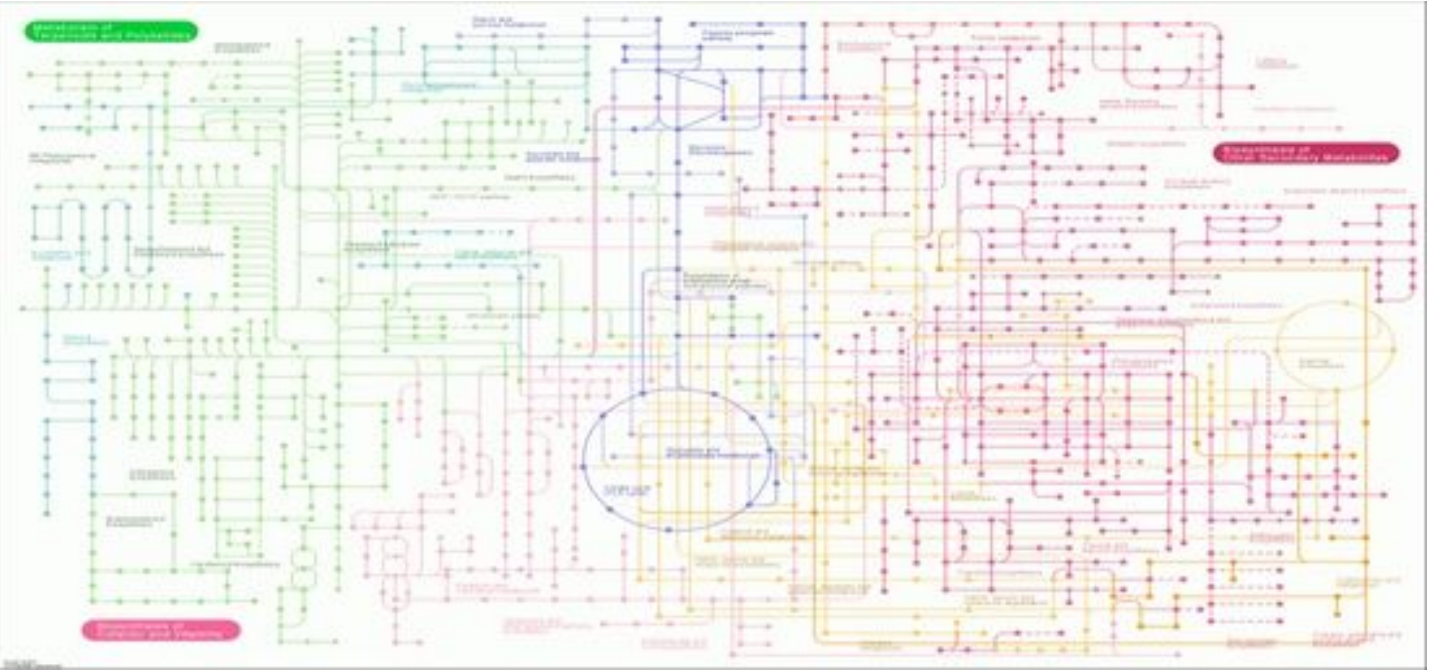

\section{Figure 2}

The secondary metabolism related to the change of CP content in Leymus chinensis 

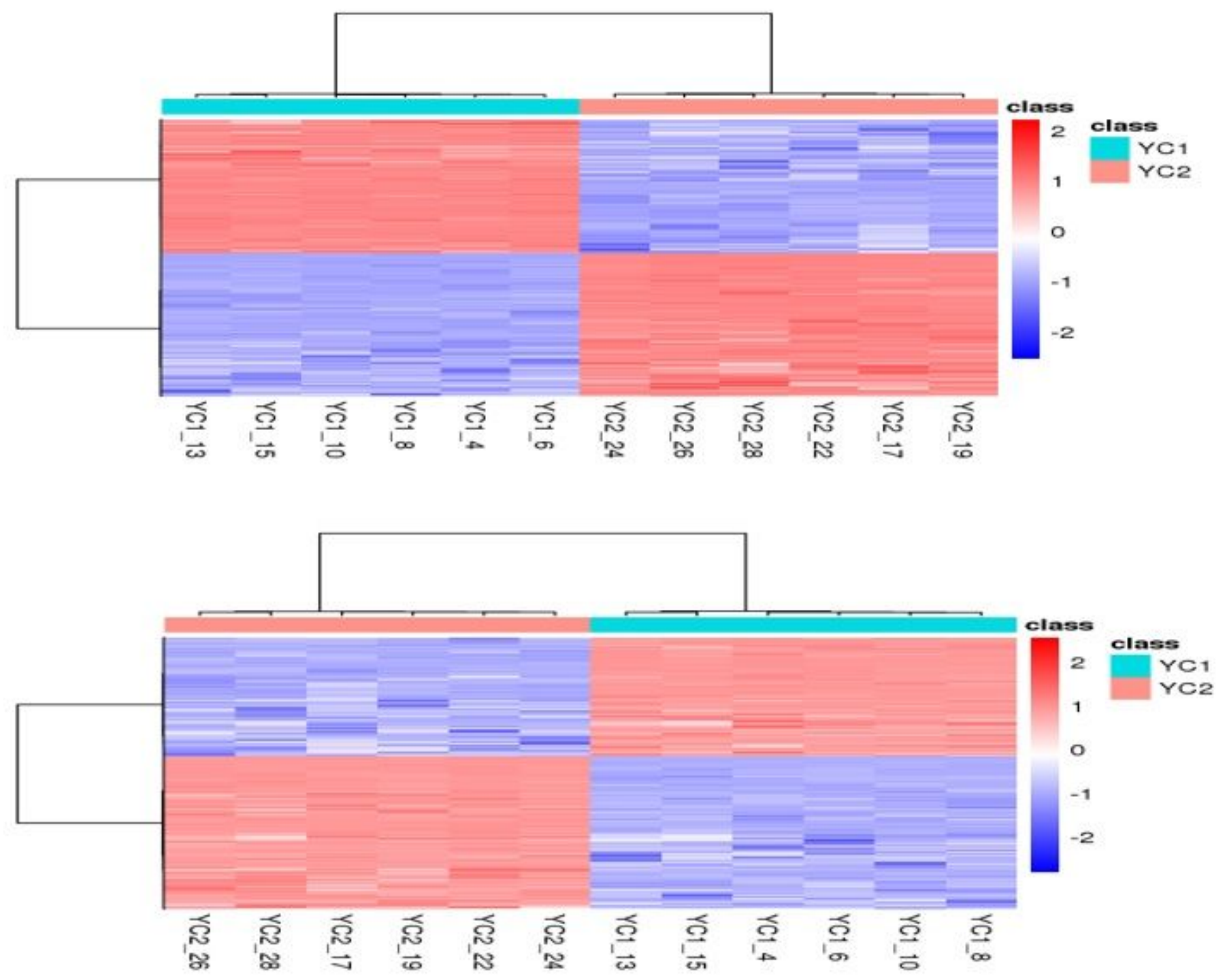

Figure 3

Hierarchical cluster heat map of differential metabolites. Hierarchical cluster heat map of significantly different metabolites during Leymus chinensis development in two harvest stages. a Positive mode, $b$ negative mode. 


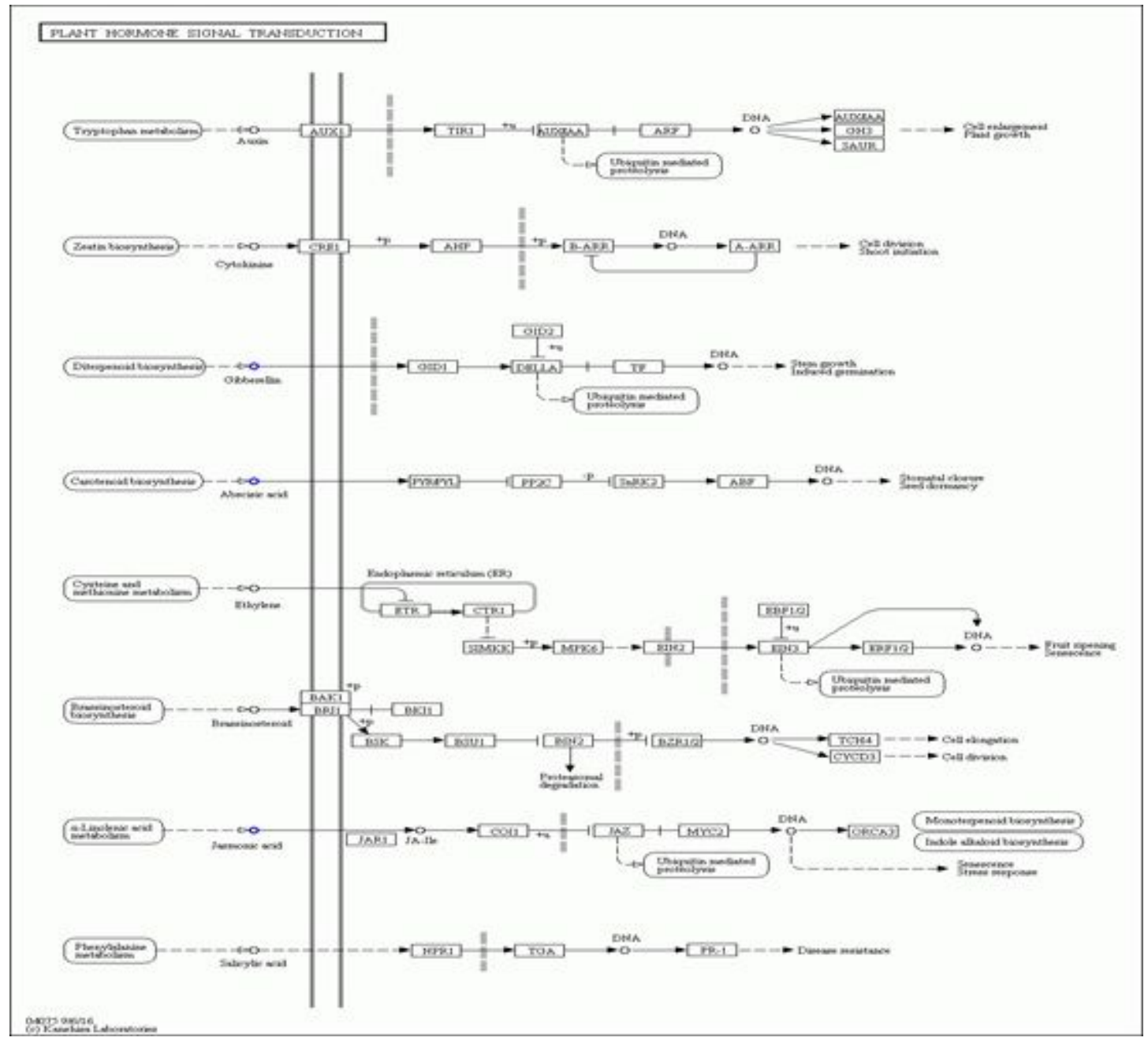

\section{Figure 4}

Leymus chinensis hormone signal transductiones
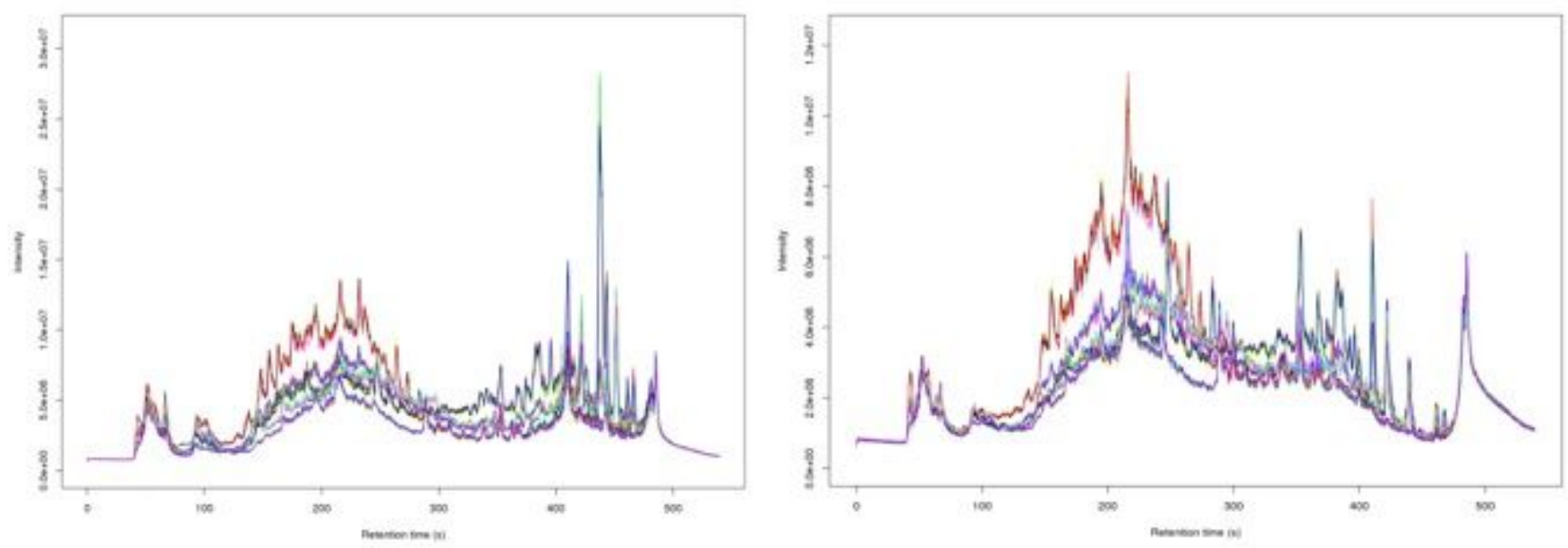

Figure 5

The TIC overlaps map of QC sample 

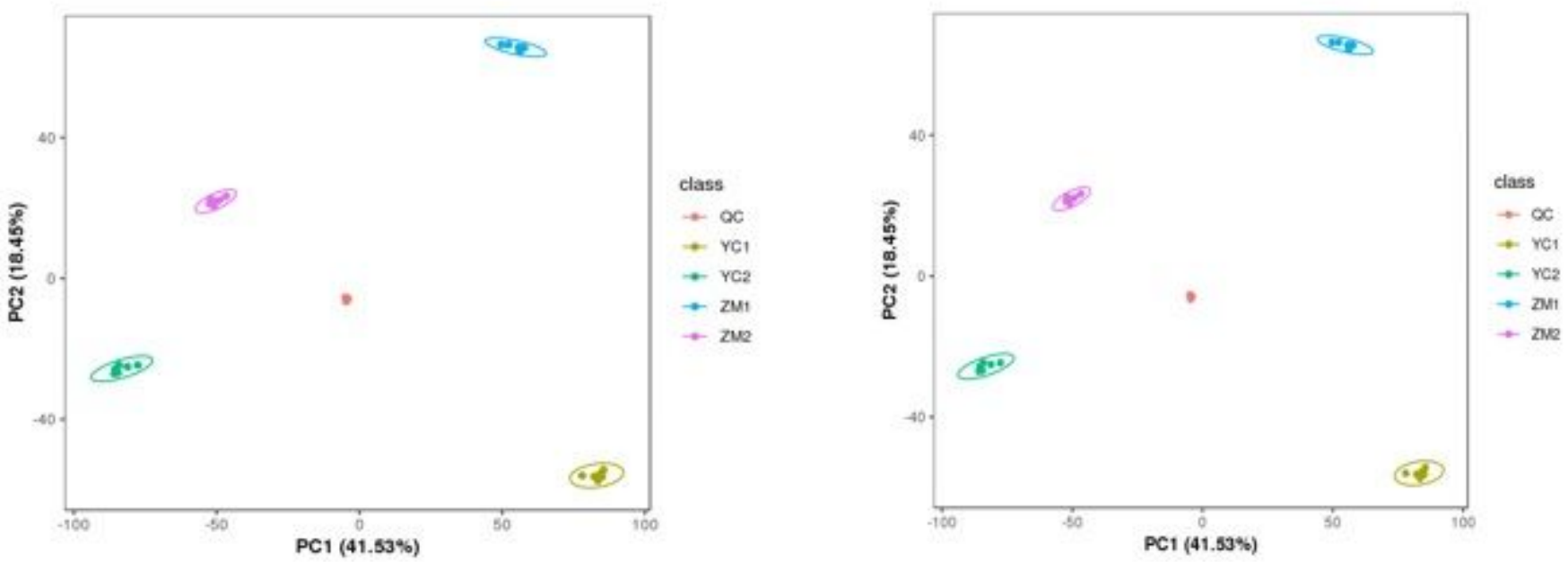

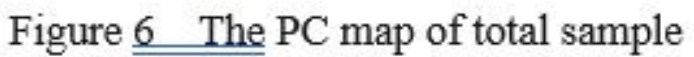

Figure 6

The PC map of total sample 